\begin{tabular}{c|c|c|} 
SELECCIONES MATEMÁTICAS \\
Universidad Nacional de Trujillo \\
ISSN: $2411-1783$ (Online) \\
2020; Vol. $7(2): 250-256$.
\end{tabular}

\title{
A new notion of convergence on ideal topological spaces
}

\section{Una nueva noción de convergencia sobre espacios topológicos ideales}

\author{
Carlos Granados (1)
}

Received, Jun. 05, 2020

Accepted, Dec. 11, 2020

How to cite this article:

Granados C. A new notion of convergence on ideal topological spaces. Selecciones Matemáticas. 2020;7(2):250-256.

http://dx.doi.org/10.17268/sel.mat.2020.02.07

\begin{abstract}
In this article, we use the notions of b-open and b-I-open sets to introduce the idea of b-I-convergence which we will denoted by b-I-convergence, we also show some of its properties. Besides, some basic properties of $b$-I-Fréchet-Urysohn space is shown. Moreover, notions related to $b$ - $I$-sequential and $b$ - $I$ sequentially are proved. Furthermore, we show some relations of $b$-I-irresolute functions between preserving $b$-I-convergence functions and $b$ - $I$-covering functions.

Keywords . $b$ - $I$-convergence, $b$ - $I$-irresolute functions, preserving $b-I$-convergence functions, $b$ - $I$-sequentially open, $b-I$-sequential spaces, $b-I$-covering functions, $b$ - $I$-Fréchet-Urysohn spaces.
\end{abstract}

\section{Resumen}

En este artículo, usamos las nociones de conjuntos b-abierto y b-I-abierto para introducir la idea de bI-convergencia la cual vamos a denotar por b-I-convergencia, también mostramos algunas de sus propiedades. Además, algunas propiedades básicas del espacio b-I-Fréchet-Urysohn son mostradas. Adicionalmente, nociones relativas a espacios pre-I-secuenciales y pre-I-secuencialmente abiertos son probadas. Además, mostramos algunas relaciones entre funciones $b-I$-irresolutas, funciones que preservan $b-I$ convergencia y funciones de $b$ - $I$-cobertura.

Palabras clave. $b-I$-convergencia, funciones $b-I$ - irresolutas, funciones que preservan $b$ - $I$-convergencia, $b-I$ secuencialmente abierto, espacios $b$ - $I$-secuenciales, funciones de $b$ - $I$-cobertura, espacios $b$ - $I$-Fréchet-Urysohn.

1. Introduction. The notion of ideal was introduced by Kuratowski in 1933 on [5], an ideal $I$ on a space $X$ is a collection of elements of $X$ which satisfies: (1) $\emptyset \in I$; (2) If $A, B \in I$ then $A \cup B \in I$; and (3) if $B \subset I$ and $A \subset B$, then $A \in I$. This notion has been grown in several concepts of general topology. In 2019, Zhou and Lin on [7] used the notion of ideal on the set $\mathbb{N}$ to extend the notion of $I$-convergence, those results were useful for the developing of this paper. On the other hand, in 1996, Andrijevi on [1] introduced the concept of $b$-open sets in a topological spaces. A subset $A$ of $(X, \tau)$ is said to be $b$-open if $A \subseteq C l(\operatorname{Int}(A)) \cup \operatorname{Int}(C l(A))$. After that in 2004, Aysegul and Gulhan on [2] presented the idea of $b$ - $I$-open sets and $b$ - $I$-continuous functions in ideal topological spaces. The $b$ - $I$-open sets were defined as: Let $(X, \tau, I)$ be an ideal topological space and let $A$ be a subset of $X$, then $A$ is said to be $b$ - $I$-open if $A \subseteq C l^{\star}(\operatorname{Int}(A)) \cup \operatorname{Int}\left(C l^{\star}(A)\right)$, where $C l^{\star}(A)=A \cup A^{\star} . A^{\star}$ is called the local function of $A$ respect to an ideal $I$ and a topological space $\tau$ which was defined by [5]. The local function of $A$ was defined as: $A^{\star}=\{x \in X: U \cap A \notin I$ for each $U \in \tau(x)\}$ where $\tau(x)=\{U \in \tau: x \in U\}$.

In this article, we took whole the notions mentioned above and we define other properties on $b-I$ convergence and we study the relation between $b$ - $I$-sequentially open and $b$ - $I$-sequential space. Moreover, we define and study some basic properties of preserving $b$ - $I$-convergence functions and $b$ - $I$-covering functions, furthermore we prove some relations with $b$ - $I$-irresolute functions. Besides, the idea of $b-I$-FréchetUrysohn space is defined.

*Universidad del Atlántico, Barranquilla-Colombia (carlosgranadosortiz@outlook. es). 
Throughout this paper, the terms $(X, \tau)$ and $(Y, \sigma)$ means topological spaces on which no separation axioms are assumed unless otherwise mentioned. Additionally, we sometimes write $X$ or $Y$ instead of $(X, \tau)$ or $(Y, \sigma)$, respectively. By other hand, $|A|$ will denote the cardinality of set $A$.

2. $b$ - $I$-convergence. We first introduce some definitions.

Definition 2.1. Let $(X, \tau)$ be a topological space, $A \subset X$ and $x \in X$. Then, $A$ is said to be $b$ neighbourhood of $x$ if and only if there exits a a b-open set $B$ such that $x \in B \subset A$.

Definition 2.2. An ideal $I \subseteq \mathbb{N}$ is said to be non-trivial, if $I=\varnothing$ and $I \neq \mathbb{N}$. A non-trivial ideal $I \subseteq K$ is called admissible if $I \supseteq\{\{n\}: n \in \mathbb{N}\}$.

Definition 2.3. Let $I$ be an ideal on $\mathbb{N}$ and $X$ be a topological space. A sequence $\left(x_{n}\right)_{n \in \mathbb{N}}$ is called $I$ convergent to a point $x \in X$, provided for any neighbourhood $V$ of $x$, it has $A_{V}=\left\{n \in \mathbb{N}: x_{n} \notin V\right\} \in I$, which is denoted by $I-\lim _{n \rightarrow \infty} x_{n}=x$ or $x_{n} \rightarrow^{I} x$.

Definition 2.4. Let $I$ be an ideal on $\mathbb{N}$ and $X$ be a topological space. A sequence $\left(x_{n}\right)_{n \in \mathbb{N}}$ is called $b$-I-convergent to a point $x \in X$, provided for any b-neighbourhood $V$ of $x$, it has $A_{V}=\left\{n \in \mathbb{N}: x_{n} \notin\right.$ $V\} \in I$, which is denoted by $b$ - $I-\lim _{n \rightarrow \infty} x_{n}=x$ or $x_{n} \rightarrow^{b I} x$ and the point $x$ is called the $b$-I-limit of the sequence $\left(x_{n}\right)_{n \in \mathbb{N}}$.

Lemma 2.1. [1] Every open set of $(X, \tau)$ is a b-open set.

Lemma 2.2. $b$-I-convergence implies I-convergence.

Proof: Let $V$ an open set of $(X, \tau)$, then by the Lemma $2.1 V$ is a $b$-open set. Since $\left\{x_{n}\right\}$ is a $b$ - $I$-convergent sequence, we have that $\left\{n \in \mathbb{N}: x_{n} \notin V\right\} \in I$. Therefore, by the Definition 2.3, $\left\{x_{n}\right\}$ is a $I$-convergent sequence.

Remark 1. The converse of the above Lemma need not be true as can be seen in the following example:

Let $\mathbb{R}$ be the set of real numbers with the usual topology and I be an admissible ideal and the sequence $\left(a_{n}\right)_{n \in \mathbb{N}}$ be defined by $a_{n}=b^{n}$, where $0<b<1$. It sees that the sequence $a_{n}=b^{n}$ is $I$-convergence to 0 , since for any open set $U$ containing 0 , the set $\left\{n \in \mathbb{N}: a_{n} \notin U\right\}$ is finite. Now, take a b-open set $V=(-1,0]$, we can see that $V$ is a b-open set since that $(-1,0] \subseteq[1,0]$. Now, we can see that the set $\left\{n \in \mathbb{N}: a_{n} \notin V\right\}$ is equal to the set of natural numbers and then the sequence $a_{n}=b^{n}$ is not b-Iconvergent to 0 .

Remark 2. Since every open set is a b-open, we proved that b-I-convergence implies I-convergence. If we would like to find an equivalent between them, $(X, \tau)$ should be a topological space with $\tau$ be the discrete topology. If $\tau$ is the discrete topology, every open set is a closed set and conversely. Let $V$ be a b-open set, then

$$
\begin{aligned}
V & \subseteq C l(\operatorname{Int}(V)) \cup \operatorname{Int}(C l(V)), \\
V & =C l(V) \cup \operatorname{Int}(V), \\
V & =V \cup V, \\
V & =V .
\end{aligned}
$$

And $V$ is a subset of $(X, \tau)$, therefore if we have a sequence $\left\{x_{n}\right\}$ which is $I$-convergent, $\left\{x_{n}\right\}$ must be $b$-I-convergent.

Remark 3. Taking into account the Remark 2, are there other conditions in which I-convergence implies b-I-convergence? This is an open problem.

Definition 2.5. Let $(X, \tau)$ be a topological space and $A \subset X$. Then, $A$ is called $b$-I-sequentially open if and only if no sequence in $X-A$ has a $b$-I-limit in $A$. i.e. sequence can not $b$-I-converge out of a $b$-I-sequentially closed set.

Definition 2.6. Let I be an ideal on $\mathbb{N}$ and $X$ be a topological space, then 
1. A subset $J$ of $X$ is said to be $b$ - $I$-closed if for each sequence $\left(x_{n}\right)_{n \in \mathbb{N}} \subseteq J$ with $x_{n} \rightarrow^{b I} x \in X$, then $x \in J$.

2. A subset $V$ of $X$ is said to be $b$ - $I$-open if $X-V$ is $b$ - $I$-closed.

3. $X$ is said to be a $b$ - $I$-sequential space if each $b$ - $I$-closed set in $X$ is closed.

Remark 4. The notion showed in the previous definition point 1 on b-I-closed set, this notion is equivalent of the notion showed by [2] about b-I-closed set.

Let $A$ be a b-I-closed and $\left\{x_{n}\right\} \subseteq A$ with $\left\{x_{n}\right\}$ a b-I-convergent to $x$. If $x \notin A$, then $x \in A^{c}$ and then we have that $\left\{x_{n}\right\} \in A^{c}$, and this is a contradiction. Therefore, $x \in A$.

The converse is proved similarly.

Definition 2.7. Let $(X, \tau)$ be a topological space. Then, $X$ is $b$ - $I$-sequential when any set $A$ is b-open if and only if it is $b$ - $I$-sequentially open.

Now, we show some results.

Lemma 2.3. (cf. [7]) Let I be an ideal on $\mathbb{N}$ and $X$ be a topological space. If a sequence $\left(x_{n}\right)_{n \in \mathbb{N}}$ $I$-converges to a point $x \in X$ and $\left(y_{n}\right)_{n \in \mathbb{N}}$ is a sequence in $X$ with $\left\{n \in \mathbb{N}: x_{n} \neq y_{n}\right\} \in I$, then the sequence $\left(y_{n}\right)_{n \in \mathbb{N}} I$-converges to $x \in X$

Lemma 2.4. (cf. [7]) Let $I \subseteq J$ be two ideals of $\mathbb{N}$. If $\left(x_{n}\right)_{n \in \mathbb{N}}$ is a sequence in a topological space $X$ such that $x_{n} \rightarrow^{I} x$, then $x_{n} \rightarrow^{J} x$.

Lemma 2.5. Let $(X, \tau)$ be a topological space. Then, $B \subset X$ is $b$-I-sequentially open if and only if every sequence with $b$ - $I$-limit in $B$ has all but finitely many terms in $B$. Where the index set of the part in $B$ of the sequence does not belong to $I$.

Proof: Suppose that $B$ is not a $b$ - $I$-sequentially open, then there is a sequence with terms in $X-B$, but $b$ - $I$-limit in $B$. Conversely, suppose that $\left(x_{n}\right)_{n \in \mathbb{N}}$ is a sequence with infinitely many terms in $X-B$ such that $b$ - $I$-converges to $y \in B$ and the index set of the part in $B$ of the sequence does not belong to $I$. Then, $\left(x_{n}\right)_{n \in \mathbb{N}}$ has a subsequence in $X-B$ that has to still converges to $y \in B$ and so $B$ is not $b$ - $I$-sequentially open.

Lemma 2.6. Let $I$ and $J$ be two ideals of $\mathbb{N}$ where $I \subseteq J$ and $X$ is a topological space. If $V \subseteq X$ is $b$-J-open, then it is b-I-open.

Proof: Let $V \subseteq X$ be $b$ - $I$-open. Then, $X-V$ is pre- $I$-closed set, so every sequence $\left(x_{n}\right)_{n \in \mathbb{N}}$ in $X-V$ with $x_{n} \rightarrow^{b I} x$, hold that $x_{n} \rightarrow^{b J} x$, by Lemma 2.4. So, $x \in X-V$ and therefore, $V$ is $b$ - $J$-open.

Corollary 2.1. Let $I$ and $J$ be two ideals of $\mathbb{N}$, where $I \subseteq J$. If a topological space $X$ is $b$-I-sequential, then it is $b$ - $J$-sequential.

Lemma 2.7. Let $I$ be an ideal on $\mathbb{N}$ and $X$ be a topological space. If a sequence $\left(x_{n}\right)_{n \in \mathbb{N}} b-I$ convergent to a point $x \in X$ and $\left(y_{n}\right)_{n \in \mathbb{N}}$ is a sequence in $X$ with $\left\{n \in \mathbb{N}: x_{n} \neq y_{n}\right\} \in I$, then the sequence $\left(y_{n}\right)_{n \in \mathbb{N}} b$-I-convergent to $x \in X$.

Proof: The proof is followed by the Lemma 2.3 and Definition 2.4.

Lemma 2.8. Let $X$ be a topological space $X, A \subset X$ and $I$ be an ideal on $\mathbb{N}$. Then, the following statements are equivalent.

1. A is b-I-open.

2. $\left\{n \in \mathbb{N}: x_{n} \in A\right\} \notin I$ for each sequence $\left(x_{n}\right)_{n \in \mathbb{N}}$ in $X$ with $x_{n} \rightarrow^{b I} x \in A$.

3. $\left|\left\{n \in \mathbb{N}: x_{n} \in A\right\}\right|=\theta$ for each sequence $\left(x_{n}\right)_{n \in \mathbb{N}}$ in $X$ with $x_{n} \rightarrow^{b I} x \in A$.

Proof: $(1) \Rightarrow(2)$ : Suppose that $A$ is a $b$ - $I$-open set of $X$ and let $\left(x_{n}\right)_{n \in \mathbb{N}}$ be a sequence in $X$ satisfying $x_{n} \rightarrow^{b I} x \in A$. Now, take $N_{0}=\left\{n \in \mathbb{N}: x_{n} \in A\right\}$. If $N_{0} \in I$, then $N_{0} \neq \mathbb{N}$ and so $A \neq X$. Now, take a point $a \in X-A$ and define the sequence $\left(y_{n}\right)_{n \in \mathbb{N}}$ in $X$ by

$$
y_{n}=\left\{\begin{array}{ccc}
a & \text { if } & n \in N_{0} \\
x_{n} & \text { if } & n \notin N_{0}
\end{array} .\right.
$$


By Lemma 2.7, the sequence $\left(y_{n}\right)_{n \in \mathbb{N}} b$ - $I$-converges to $x$. We can see that $X-A$ is $b$ - $I$-closed and $\left(y_{n}\right)_{n \in \mathbb{N}} \subseteq X-A$, in consequence $x \in X-A$ and this is a contradiction. Therefore, $N_{0} \notin I$.

The implication $(2) \Rightarrow(3)$ it follows form the notion that the ideal $I$ is admissible.

Now, it shows the following implication. $(3) \Rightarrow(1)$ : Let $A$ not be $b-I$-open in $X$. Then, $X-A$ is no $b$ - $I$-closed and there is a sequence $\left(x_{n}\right)_{n \in \mathbb{N}} \subseteq X-A$ with $x_{n} \rightarrow^{b I} x \in A$ and this is a contradiction.

Theorem 2.1. Every b-I-sequential space is hereditary with respect to b-I-open (b-I-closed) subspaces.

Proof: Let $X$ be a $b$ - $I$-sequential space. Now, suppose that $A$ is a $b-I$-open set of $X$. Then, $A$ is $b$-open in $X$. Now, we can see that $A$ is $b-I$-sequential. Let $V$ be a pre- $I$-open set in $A$, thus $V$ is $b$-open in $X$. Indeed, by the Definition 2.7, if we show that $V$ is $b$ - $I$-open in $X$, it will be sufficient.

Now, suppose that there is a point $y \in X-V$ and take an arbitrary $x \in V$ and a sequence $\left(x_{n}\right)_{n \in \mathbb{N}} \subseteq X$ with $x_{n} \rightarrow^{b I} x$ in $X$. Since, $A$ is $b$-open in $X$ and $x \in A$, the set $\left\{n \in \mathbb{N}: x_{n} \notin A\right\} \in I$. We define the sequence $\left(y_{n}\right)_{n \in \mathbb{N}}$ in $X$ by

$$
y_{n}=\left\{\begin{array}{ccc}
x_{n} & \text { if } & x_{n} \in A \\
y & \text { if } & x_{n} \notin A
\end{array} .\right.
$$

By the Lemma 2.7, the sequence $\left(y_{n}\right)_{n \in \mathbb{N}} b$-I-converges to $x$. Since $\left|\left\{n \in \mathbb{N}: x_{n} \notin V\right\}\right|=\mid\{n \in \mathbb{N}$ : $\left.y_{n} \notin V\right\} \mid$ and by the Lemma $2.8, V$ is $b$ - $I$-open in $X$.

Now, let $A$ be a $b$ - $I$-closed set of $X$. Then, $A$ is pre-closed in $X$. For any $b$ - $I$-closed set $J$ of $A$. It has to show that $J$ is $b$-closed in $X$. Since $X$ is a $b-I$-sequential space, it is enough that $J$ is $b$ - $I$-closed in $X$. Hence, let $\left(x_{n}\right)_{n \in \mathbb{N}}$ be an arbitrary sequence in $J$ with $x_{n} \rightarrow^{b I} x \in X$. It obtains that $x \in J$. Indeed, since $A$ is $b$-closed, it has that $x \in A$ and so $x \in J$ since $J$ is a $b$ - $I$-closed set of $A$.

Theorem 2.2. $b$-I-sequential spaces are preserved by topological sums.

Proof: Let $\left\{X_{\delta}\right\}_{\delta \in \Delta}$ be a family of $b$ - $I$-sequential spaces. Take $X=\bigoplus_{\delta \in \Delta} X_{\delta}$, being the topological sum of $\left\{X_{\delta}\right\}_{\delta \in \Delta}$. Now, it will show that the topological sum is a $b$ - $I$-sequential space. Let $J$ be a $b$ - $I$-closed set in $X$. For each $\delta \in \Delta$, since $X_{\delta}$ is $b$-closed in $X, J \cap X_{\delta}$ is $b$ - $I$-closed in $X$. We can see that $J \cap X_{\delta} \subseteq X_{\delta}$ and $J \cap X_{\delta}$ is $b$ - $I$-closed in $X_{\delta}$. By the assumption, it has that $J \cap X_{\delta}$ is $b$-closed in $X_{\delta}$. By the definition of topological sums, it gets that $J$ is $b$-closed in $X$. Therefore, the topological sum $X$ is a $b$ - $I$-sequential space.

Remark: The union of a family of $b-I$-open sets of a topological space is $b$ - $I$-open. Therefore, the intersection of finitely many sequentially $b-I$-open sets is sequentially $b-I$-open.

Definition 2.8. (cf. [7]) Let I be an ideal on $\mathbb{N}$ and $A$ be a subset of a topological space $X$. A sequence $\left(x_{n}\right)_{n \in \mathbb{N}}$ in $X$ is I-eventually in $A$ if there is $B \in I$ such that, for all $n \in \mathbb{N}-B, x_{n} \in A$.

Proposition 2.1. Let I be a maximal ideal on $\mathbb{N}$ and $X$ be a topological space. Then, $A$ is a subset of $X$ where $A$ is $b$-I-open if and only if each pre-I-convergent sequence in $X$, converging to a point of $A$ is I-eventually in $A$.

Proof: Let $A$ be a $b$ - $I$-open and $x_{n} \rightarrow^{b I} x \in A$. Since $I$ is maximal, by the Lemma $2.8, B=\{n \in \mathbb{N}$ : $\left.x_{n} \notin A\right\} \in I$. Therefore, for each $n \in \mathbb{N}-B, x_{n} \in A$, i.e., the sequence $\left(x_{n}\right)_{n \in \mathbb{N}}$ is $I$-eventually in $A$.

Theorem 2.3. Let $I$ be an ideal of $\mathbb{N}$ and $X$ be a topological space. If $V, W$ are two b-I-open sets of $X$, then $V \cap W$ is $b$-I-open.

Proof: It will be shown that every $b$ - $I$-convergent sequence converging to a point in $V \cap W$ is $I$ eventually in it. Let $\left(x_{n}\right)_{n \in \mathbb{N}}$ be a sequence in $X$ such that $x_{n} \rightarrow^{b I} x \in V \cap W$. There are $A, S \in I$ such that for each $n \in \mathbb{N}-A, x_{n} \in V$ and for each $n \in \mathbb{N}-S, x_{n} \in W$. Since $A \cup S \in I$ and for each $n \in \mathbb{N}-(A \cup S), x_{n} \in V \cap W$, therefore $V \cap W$ is a $b$ - $I$-open set.

\section{Further properties.}


3.1. $b$ - $I$-irresolute functions. In this part, it is introduced $b$ - $I$-irresolute functions and it shows some relations among continuous and $b-I$-continuous functions.

Definition 3.1. (cf. [3]) Let $f:(X, \tau) \rightarrow(Y, \sigma)$ be a functions. $f$ is called sequentially continuous provided $V$ is sequentially open in $Y$, then $f^{-1}(V)$ is sequentially open in $X$.

Definition 3.2. Let $I$ be an ideal on $\mathbb{N},(X, \tau),(Y, \sigma)$ be a topological spaces and $f:(X, \tau) \rightarrow(Y, \sigma)$ be a function, then.

1. $f$ is said to be preserving $b$-I-convergence provided for each sequences $\left(x_{n}\right)_{n \in \mathbb{N}}$ in $X$ with $x_{n} \rightarrow^{b I}$ $x$, the sequence $\left(f\left(x_{n}\right)\right)_{n \in \mathbb{N}} b$ - $I$-converges to $f(x)$.

2. $f$ is said to be b-I-irresolute if for each b-I-open $V$ in $Y$, then $f^{-1}(V)$ is b-I-open in $X$ (cf. [2]).

Lemma 3.1. (cf. [2]) Every b-I-irresolute function is b-I-continuous.

Theorem 3.1. Let $f:(X, \tau) \rightarrow(Y, \sigma)$ be a function. If $f$ is continuous, then $f$ preserves $b-I$ convergence.

Proof: Suppose that $f$ is continuous and let $\left(x_{n}\right)_{n \in \mathbb{N}}$ be a sequence in $X$ such that $x_{n} \rightarrow^{b I} x \in X$. Now, let $V$ be an arbitrary semi-neighbourhood of $f(x)$ in $Y$. Since $f$ is continuous, $f^{-1}(V)$ is a semineighbourhood of $x$. Therefore, it has that $\left\{n \in \mathbb{N}: x_{n} \notin f^{-1}(V)\right\} \in I$. We can see that $\left\{n \in \mathbb{N}: f\left(x_{n}\right) \notin\right.$ $V\}=\left\{n \in \mathbb{N}: x_{n} \notin f^{-1}(V)\right\}$. This implies that $\left\{n \in \mathbb{N}: f\left(x_{n}\right) \notin V\right\} \in I$. Hence, $f\left(x_{n}\right) \rightarrow^{b I} f(x)$.

Theorem 3.2. Let $f:(X, \tau) \rightarrow(Y, \sigma)$ be a function. If $f$ preserves $b$ - $I$-convergence, then $f$ is $b$ - $I$ irresolute.

Proof: Suppose that $f$ preserves $b$ - $I$-convergence and $J$ is an arbitrary $b$ - $I$-closed set in $Y$. Let $\left(x_{n}\right)_{n \in \mathbb{N}}$ be a sequence in $f^{-1}(J)$ such that $x_{n} \rightarrow^{b I} x \in X$. By the assumption, it has that $f\left(x_{n}\right) \rightarrow^{b I} f(x)$. Since $\left(f\left(x_{n}\right)\right)_{n \in \mathbb{N}} \subseteq J$ and $J$ is $b$ - $I$-closed in $Y$, hence $f(x) \in J$, i.e., $x \in f^{-1}(J)$. Therefore, $f^{-1}(J)$ is $b$ - $I$-closed in $X$ and then $f$ is $b$ - $I$-irresolute.

Proposition 3.1. Let $f:(X, \tau) \rightarrow(Y, \sigma)$ be a function. If $f$ preserves $b$-I-convergence, then $f$ is $b$-I-continuous.

Proof: The proof is followed by the Lemma 3.1 and Theorem 3.2.

Theorem 3.3. Let I be anideal on $\mathbb{N}$. Then, a function $f:(X, \tau) \rightarrow(Y, \sigma)$ is b-I-irresolute if and only if it preserves $b-I$-convergent sequences.

Proof: Assume that $f$ is $b$ - $I$-irresolute and a sequence $x_{n} \rightarrow^{b I} x$ in $X$. It has to show that $f\left(x_{n}\right) \rightarrow^{b I}$ $f(x)$ in $Y$. Now, let $V$ a semi-neighbourhood of $f(x)$. Then, $x \in f^{-1}(V)$ is $b-I$-open in $X$, because $V$ is $b$ - $I$-open in $Y$. Hence, there is $B \in I$ such that for all $n \in \mathbb{N}-B, x_{n} \in f^{-1}(V)$. This means that for all $n \in \mathbb{N}-B, f\left(x_{n}\right) \in V$. Therefore, $\left\{n \in \mathbb{N}: f\left(x_{n}\right) \notin V\right\} \in I$ and hence $f\left(x_{n}\right) \rightarrow^{b I} f(x)$.

Theorem 3.4. Let $X$ be a b-I-sequential space and $f:(X, \tau) \rightarrow(Y, \sigma)$ be a function. Then, the following statements are equivalent.

1. $f$ is continuous.

2. $f$ preserves $b$ - $I$-convergence.

3. $f$ is $b$-I-irresolute.

Proof: $(1) \Leftrightarrow(2)$ was proved in the Theorems 3.1 and 3.2.

$(3) \Rightarrow(1)$ : Let $f$ be $b$ - $I$-irresolute and $J$ be an arbitrary $b$-closed set in $Y$. Then, $J$ is $b$ - $I$-closed in $Y$. Since $f$ is $b$ - $I$-irresolute, $f^{-1}(J)$ is $b$ - $I$-closed in $X$. By assumption, it has that $f^{-1}(J)$ is $b$-closed in $X$. Therefore, $f$ is continuous.

Proposition 3.2. Let $f:(X, \tau) \rightarrow(Y, \sigma)$ be a function and $X$ be a $b$-I-sequential space. Then, the following statements are equivalent.

1. $f$ is continuous.

2. $f$ is b-I-continuous. 
Proof: The proof is followed by the Proposition 3.1 and Theorem 3.4.

Lemma 3.2. Let $X$ be a $b$-I-sequential space, then the function $f:(X, \tau) \rightarrow(Y, \sigma)$ is continuous if and only if it is sequentially continuous.

Proof: Let $X$ be a $b$ - $I$-sequential space, then every $b$ - $I$-closed set is closed, by [3] who proved that $f$ is continuous if and only if $f$ is sequentially continuous, indeed we have completed the proof.

Corollary 3.1. Let $X$ be a $b$-Isequential space and for a function $f:(X, \tau) \rightarrow(Y, \sigma)$ the following statements are equivalent.

1. $f$ is continuous.

2. $f$ preserves $b-I$-convergence.

3. $f$ is b-I-continuous.

4. $f$ is sequentially continuous.

Proof: $(1) \Leftrightarrow(2) \Leftrightarrow(3)$ was proved in the Theorem 3.4, by the Lemma 3.2, we have $(1) \Leftrightarrow(4)$.

Lemma 3.3. Let $f:(X, \tau) \rightarrow(Y, \sigma)$ be a function and $X$ be a $b$-I-sequential space. Then, the following statements are equivalent.

1. $f$ is sequentially continuous.

2. $f$ is b-I-continuous.

Proof: The proof is followed by the Proposition 3.2 and Corollary 3.1.

3.2. $b$ - $I$-irresolute and $b$ - $I$-covering functions. Continuity and sequentially continuity are ones of the most important tools for studying sequential spaces on [6]. In this part, it is defined the concept of $b-I$-covering functions and it is shown some of their properties.

Definition 3.3. (cf. [3]) Let $f:(X, \tau) \rightarrow(Y, \sigma)$ be a topological space. Then, $f$ is said to be sequentially continuous provided $f^{-1}(V)$ is sequentially open in $X$, then $V$ is sequentially open in $Y$.

Definition 3.4. (cf. [3]) Let $f:(X, \tau) \rightarrow(Y, \sigma)$ be a topological space. Then, $f$ is said to be sequence-covering if, whenever $\left(y_{n}\right)_{n \in \mathbb{N}}$ is a sequence in $Y$ covering to $y$ in $Y$, there exits a sequence $\left(x_{n}\right)_{n \in \mathbb{N}}$ of points $x_{n} \in f^{-1}\left(y_{n}\right)$ for all $n \in \mathbb{N}$ and $x \in f^{-1}(y)$ such that $x_{n} \rightarrow x$.

Definition 3.5. Let $f:(X, \tau) \rightarrow(Y, \sigma)$ be a function. Then, $f$ is said to be $b$ - $I$-covering if, whenever $\left(y_{n}\right)_{n \in \mathbb{N}}$ is a sequence in $Y, b$-I-converging to $y$ in $Y$, there exits a sequence $\left(x_{n}\right)_{n \in \mathbb{N}}$ of points $x_{n} \in f^{-1}\left(y_{n}\right)$ for all $n \in \mathbb{N}$ and $x \in f^{-1}(y)$ such that $x_{n} \rightarrow^{b I} x$.

Theorem 3.5. Every $b$ - $I$-covering function is $b$-I-irresolute.

Proof: Let $f:(X, \tau) \rightarrow(Y, \sigma)$ be a function and $f$ be a $b$ - $I$-covering function. Now, assume that $V$ is a non- $b$ - $I$-closed in $Y$. Then, there exits a sequence $\left(y_{n}\right)_{n \in \mathbb{N}} \subseteq V$ such that $y_{n} \rightarrow^{b I} y \notin V$. Since $f$ is $b$ - $I$-covering, there exits a sequence $\left(x_{n}\right)_{n \in \mathbb{N}}$ of points $x_{n} \in f^{-1}\left(y_{n}\right)$ for all $n \in \mathbb{N}$ and $x \in f^{-1}(y)$ such that $x_{n} \rightarrow^{b I} x$. Now, we can see that $\left(x_{n}\right)_{n \in \mathbb{N}} \subseteq f^{-1}(V)$ and so $x \notin f^{-1}(V)$, therefore $f^{-1}(V)$ is non- $b$ - $I$-closed. In conclusion, $f$ is $b$ - $I$-irresolute.

Theorem 3.6. Let $f:(X, \tau) \rightarrow(Y, \sigma)$ be a function. Then, the following statements hold.

1. If $X$ is a $b$-I-sequential space and $f$ is continuous, then $Y$ is a $b$ - $I$-sequential space and $b-I$ irresolute.

2. If $Y$ is a $b$ - $Y$-sequential space and $f$ is $b$-I-irresolute, then $f$ is continuous.

Proof:

1. Let $X$ be a $b$ - $I$-sequential space and $f$ be continuous. Suppose that $V$ is $b$ - $I$-open in $Y$. Since $f$ is a continuous function and $X$ is a $b$ - $I$-sequential space, take an arbitrary sequence $\left(x_{n}\right)_{n \in \mathbb{N}} \subseteq X$ such that $x_{n} \rightarrow^{b I} x \in f^{-1}(V)$ in $X$. Since $f$ is a continuous function, by the Theorem 3.1, $f\left(x_{n}\right) \rightarrow^{b I} f(x) \in V$. Now, since $V$ is pre- $I$-open in $Y$ and by the Lemma 2.8 , it has that $\left|\left\{n \in \mathbb{N}: f\left(x_{n}\right) \in V\right\}\right|=\theta$, i.e., $\left|\left\{n \in \mathbb{N}: x_{n} \in f^{-1}(V)\right\}\right|=\theta$. Therefore, $f^{-1}(V)$ is $b$ - $I$-open in $X$. 
Now, assume that $V \subseteq Y$ such that $f^{-1}(V)$ is $b$ - $I$-open in $X$. Then, $f^{-1}(V)$ is a open set of $X$ since $X$ is $b$ - $I$-sequential space. as well know that $f$ is continuous, then $V$ is open in $Y$. Hence, $f$ is continuous.

2. Let $Y$ be a $b$ - $I$-sequential space and $f$ be $b$ - $I$-irresolute. If $f^{-1}(V)$ is a open set of $X$, then $f^{-1}(V)$ is a $b$ - $I$-open set of $X$. Since $f$ is $b$ - $I$-irresolute, $V$ is a $b$ - $I$-open set of $Y$. Now, we know that $Y$ is a $b$ - $I$-sequential space and so $V$ is an open set of $Y$. Therefore, $f$ is continuous.

By the Theorems 3.4 and 3.6 it is had the following result.

Corollary 3.2. Let $f:(X, \tau) \rightarrow(Y, \sigma)$ be a function, then $f$ is continuous if and only if $f$ is $b-I$ irresolute and $Y$ is a b-I-sequential space.

3.3. $b$ - $I$-Fréchet-Urysohn spaces. A topological space $X$ is said to be Fréchet-Urysohn (cf. [4]) if for each $A \subseteq X$ and each $x \in C l(A)$, there is a sequence in $A$ converging to the point $x$ in $X$. Now, in this part, it introduces the notion of $b$ - $I$-Fréchet-Urysohn and it shows a short result.

Definition 3.6. Let $(X, \tau)$ be a topological space. Then, $X$ is said to be b-I-Fréchet-Urysohn or simply $b$ - $I$-FU, if for each $A \subseteq X$ and each $x \in b C l(A)$, there exits a sequence in $A b$ - $I$-converging to the point $x \in X$.

Lemma 3.4. For two ideals $I$ and $J$ on $\mathbb{N}$ where $I \subseteq J$, if $X$ is a $b$ - $I$-FU-space, then it is a $b$ - $J-F U$ space.

Proof: Let $A$ be a subset of $X$ and $x \in b C l(A)$. Since $X$ is a $b-I-F U$-space, then there exits a sequence $\left(x_{n}\right)_{n \in \mathbb{N}}$ in $A$ such that $x_{n} \rightarrow^{b I} x$, in consequence $x_{n} \rightarrow^{b I} x$ in $X$, and so $X$ is $b-J$ - $F U$-space.

Theorem 3.7. Let $(X, \tau)$ be a topological space. Then, $X$ is a $b$-I-FU-space, then $X$ is a $b-I$ sequential space.

Proof: Let $\left\{A_{\delta}: \delta \in \Delta\right\}$ be a family of $b$ - $I$-closed subsets of $X$ where $\delta \in \Delta \in X$, since $X$ is a $b$ - $I$ $F U$-space, by the Definition 3.6 $A_{\delta} \subseteq X$ and each $x \in b C l\left(A_{\delta}\right)$. Now, since $A_{\delta}$ is $b$ - $I$-closed $b C l\left(A_{\delta}\right)=$ $A_{\delta} \in C l(A)$, but by the Definition 3.6, there exits a $b$ - $I$-converging to the point $x \in b C l(A) \in C l(A) \in X$, therefore $\left\{A_{\delta}: \delta \in \Delta\right\}$ is a closed set of $X$. In consequence $X$ is a $b$ - $I$-sequential space.

ORCID and License

Carlos Granados https: / / orcid.org/0000-0002-7754-1468

This work is licensed under the Creative Commons Attribution-NoComercial-ShareAlike 4.0.

\section{References}

[1] Andrijevi D. On $b$-open sets. Mat. Vesnik. 1996; 48:59-64.

[2] Aysegul G, Gulhan A. b-I-open sets and descomposition of continuity via idealizacion. Processing of IMM of NAS of Azerbaijan. 2004; 27-32.

[3] Boone J, Siwiec F. Sequentially quotient mappings. Czechoslov. Math. J. 2018; 26:174-182.

[4] Franklin S. Spaces in which sequences suffice. Fund. Math.. 1965; 57:107-115.

[5] Kuratowski K. Topologie. Monografie Matematyczne tom 3. Warszawa: PWN-ploish Scientific Publishers. 1933.

[6] Lin S, Yun Z. Generalized metric spaces and mapping. Atlantis Studies in Mathematics. 2016; 6.

[7] Zhou X, Lin S. On topological spaces defined by I-convergence. Bulletin of the Iranian Math. Society; 2019. 